\title{
Extension of the Flux Reconstruction Method to High-Reynolds Number RANS Simulations around High-Lift Devices*
}

\author{
Koji MIYAJI ${ }^{\dagger}$ and Rei NAGASAWA \\ Yokohama National University, Yokohama, Kanagawa 240-8501, Japan
}

\begin{abstract}
Spatially high-order flow simulations are conducted for high-Reynolds number flows around two-dimensional high-lift devices. The method uses a 'flux-reconstruction (FR) approach' that is applicable to unstructured quadrilateral or hexahedral grids. This is the first study focused on solving Reynolds-averaged Navier-Stokes equations coupled with $k$ - $\omega$ turbulence model equations using the FR method. The performance of the turbulence model in the high-order scheme is first verified using standard benchmark problems. The flow around the three-element, high-lift airfoil known as NHLP/ L1T2 is then tried. Simulations from second-order (solution polynomial degree 1 , or $p=1$ ) to fourth-order $(p=3$ ) accuracy all predicted the surface pressure well, while the total pressure distribution in the wake was captured well by $p=2$ and $p=3$ simulations. The effects of new wall boundary conditions and minimum cell size are qualitatively discussed.
\end{abstract}

Key Words: CFD, High-Order Method, Turbulence Model

\section{Nomenclature}

A: parameter in the wall-boundary condition of $\omega$

$F$ : flux vector

$g$ : flux correction function

$J$ : Jacobian matrix of transformation

$k$ : turbulent kinetic energy

$M$ : shape function in the standard element

$N$ : number of solution points in each direction

$Q$ : vector of conserved variables

$R e$ : Reynolds number

$S$ : vector of source terms

$S_{i j}$ : mean strain rate tensor

$u$ : flow velocity

$p$ : polynomial degrees

$\boldsymbol{x}$ : coordinates in the physical space

$y_{1}$ : distance to the 1 st grid point off the wall

$\mu$ : molecular viscosity

$\mu_{t}$ : eddy viscosity

$\rho$ : flow density

$\tau_{i j}$ : Reynolds stress tensor

$\omega$ : specific dissipation rate

$\tilde{\omega}$ : transformed $\omega$, or, $\tilde{\omega}=\ln \omega$

$\tilde{\omega}_{r}: \tilde{\omega}$ bound by realizability conditions

$\xi$ : coordinates in the computational space on the standard element

$\phi$ : basis of Lagrange polynomial

Subscripts

$e$ : element-wise continuous polynomial

$i$ : $i$-th component of the vector

$j / k: j$-th $/ k$-th solution point in $\xi / \eta$ direction

$L / R$ : left/right correction function

(C) 2017 The Japan Society for Aeronautical and Space Sciences *Received 7 October 2015; final revision received 17 June 2016; accepted for publication 16 August 2016.

†Corresponding author, miyaji@ynu.ac.jp
+ : flux at the right or the top face of the cell

-: flux at the left or the bottom face of the cell

Superscripts

com: common numerical flux at cell boundary

+ : boundary layer property in the wall unit

\section{Introduction}

With the increasing speed and size of commercial aircraft, better performance of high-lift devices (HLD) is required to keep the take-off/landing distances in an appropriate range. HLDs are key to economical efficiency as well as environmental compatibility because they contribute to the ability to increase payloads and can be the main source of aerodynamic noise at high angles-of-attack. Computational fluid dynamics (CFD) is routinely used today for the aerodynamic design of HLDs, but reliability is still not as high as during cruise conditions, due to the complexity of the configuration and flow. The boundary-layer transition of the flow, flow separation and the interaction of the wake and the downstream element must be properly predicted.

The authors participated in a CFD workshop for HLDs ${ }^{1)}$ organized by the Japan Aerospace Exploration Agency (JAXA) in 2006-2007 and carried out simulations using an unstructured finite-volume method that can be used for actual three-dimensional aircraft configurations. The model was a wing-body configuration with slats, flaps, flap-track fairings and an engine nacelle arranged around the main wing. Our results showed large discrepancies of the lift and drag from the experiment, and we never obtained satisfactory results. However, one effective trial in our simulation used the same second-order spatial accuracy in the convective term of the turbulence model as in the Reynolds-averaged NavierStokes (RANS) equations. It is said that the source term is a dominant factor in turbulence models and the convection term is less important, but the simulation on relatively coarse 
tetrahedral grids may have suffered from numerical dissipation by the first-order scheme.

In recent years, high-order accurate methods for unstructured grid CFD are being rigorously investigated and great advances have been made. The discontinuous Galerkin $(\mathrm{DG})^{2,3)}$ method has a history of nearly 20 years, and is now one of the most widely used methods with credibility. Other approaches more recently proposed to enhance computational efficiencies are the spectral difference $(\mathrm{SD})^{4)}$ and flux reconstruction $(\mathrm{FR})^{5,6)}$ methods. The FR proposed by Huynh gives a unifying framework of the high-order methods including the DG and SD by changing the 'flux correction function.' The authors have developed a FR method on unstructured hexahedral grids and showed the effectiveness of the scheme for resolved and under-resolved vortical flows. The former is a low-Reynolds number laminar flow around a delta wing, ${ }^{7)}$ and the latter is a transitional flow around an airfoil at a Reynolds number of $6 \times 10^{4}$ using implicit largeeddy simulations (ILES). ${ }^{8)}$ The present study further extends the FR to high-Reynolds number flows using RANS and turbulence model equations. For the practical use of high-order CFD for aircraft aerodynamic designs, the computational efficiency and practicality of turbulence models should be exploited, although high-order methods would fully manifest performance for LES. We use the $k-\omega$ two-equation model of Wilcox, ${ }^{9)}$ which we have sufficient experience with using the finite-volume method. This is the first study of solving RANS with the $k-\omega$ model using the FR method. We actually found no other studies using the same model, possibly due to numerical difficulties peculiar to the model. We first show verification of the method developed using a flat-plate boundary layer and a single airfoil at a high angle-of-attack. We then show the results for a two-dimensional, threeelement HLD known as NHLP/L1T2.

\section{Governing Equations}

The governing equations are the compressible NavierStokes equations and $k-\omega$ turbulence model equations. They are written in a vector form as,

$$
\partial Q / \partial t+\partial F_{i} / \partial x_{i}=S
$$

where $Q, F_{i}$ and $S$ are the vector of conserved variables, fluxes and source terms, respectively. Note the elements of $S$ are not 0 only for the $k-\omega$ equations. One of the unknown variables $\omega$, the specific dissipation rate, varies rapidly by almost five orders of magnitude in the narrow region from the wall surface to the outer edges of the boundary layers. This can be a cause of numerical instability in high-order methods. Therefore, as proposed by Bassi et al. ${ }^{2)}$ for implementing the model to the DG, a transformed variable $\tilde{\omega}=\ln \omega$ is used as a working variable. The elements of vectors in Eq. (1) for the $k-\tilde{\omega}$ equations converted from the original $k-\omega$ are written as follows. The elements for the RANS are commonly found in, for example, Wilcox ${ }^{9)}$ and Ref. 10).

$$
\begin{aligned}
& Q=\left[\begin{array}{c}
\rho k \\
\rho \tilde{\omega}
\end{array}\right] \\
& F_{i}=\left[\begin{array}{c}
\rho u_{i} k-\left(\mu+\sigma^{*} \bar{\mu}_{t}\right) \frac{\partial k}{\partial x_{i}} \\
\rho u_{i} \tilde{\omega}-\left(\mu+\sigma \bar{\mu}_{t}\right) \frac{\partial \tilde{\omega}}{\partial x_{i}}
\end{array}\right] \\
& S=\left[\begin{array}{c}
\tau_{i j} \frac{\partial u_{i}}{\partial x_{j}}-\beta^{*} \rho \bar{k} \exp \left(\tilde{\omega}_{r}\right) \\
\frac{\alpha}{\bar{k}} \tau_{i j} \frac{\partial u_{i}}{\partial x_{j}}-\beta \rho \exp \left(\tilde{\omega}_{r}\right)+\left(\mu+\sigma \bar{\mu}_{t}\right) \frac{\partial \tilde{\omega}}{\partial x_{j}} \frac{\partial \tilde{\omega}}{\partial x_{j}}
\end{array}\right] \\
& \tau_{i j}=2 \bar{\mu}_{t}\left[S_{i j}-\frac{1}{3} \frac{\partial u_{k}}{\partial x_{k}} \delta_{i j}\right]-\frac{2}{3} \rho \bar{k} \delta_{i j} \\
& S_{i j}=\frac{1}{2}\left(\frac{\partial u_{i}}{\partial x_{j}}+\frac{\partial u_{j}}{\partial x_{i}}\right) \\
& \bar{\mu}_{t}=\alpha^{*} \rho \bar{k} \exp \left(-\tilde{\omega}_{r}\right) \\
& \bar{k}=\max (0, k)
\end{aligned}
$$

where $\rho$ is the flow density, $u_{i}$ is the $i$-th component of the velocity vector and $\mu$ is the laminar (molecular) viscosity. The closure coefficients reported by Wilcox ${ }^{9)}$ are as follows.

$$
\begin{array}{ll}
\alpha=5 / 9, & \alpha^{*}=1, \quad \beta=3 / 40, \quad \beta^{*}=9 / 100, \\
\sigma=1 / 2, & \sigma^{*}=1 / 2
\end{array}
$$

In Eqs. (4) and (7), $\tilde{\omega}_{r}$ is another important mean for the numerical stability defined by Bassi et al. ${ }^{2)}$ as $\tilde{\omega}_{r}=\max \left(\tilde{\omega}, \tilde{\omega}_{r 0}\right)$, which is determined by the lower bound, $\tilde{\omega}_{r 0}$, satisfies the 'realizability condition' of the normal (Eq. (10)) and shear (Eq. (11)) Reynolds stresses.

$$
\begin{gathered}
\exp \left(\tilde{\omega}_{r 0}\right)-3 \alpha^{*}\left(S_{i i}-\frac{1}{3} \frac{\partial u_{k}}{\partial x_{k}}\right) \geq 0, \quad i=1,2,3 \\
\left\{\exp \left(\tilde{\omega}_{r 0}\right)\right\}^{2}-3 \alpha^{*}\left(S_{i i}+S_{j j}-\frac{2}{3} \frac{\partial u_{k}}{\partial x_{k}}\right) \exp \left(\tilde{\omega}_{r 0}\right) \\
+9 \alpha^{* 2}\left[\left(S_{i i}-\frac{1}{3} \frac{\partial u_{k}}{\partial x_{k}}\right)\left(S_{j j}-\frac{1}{3} \frac{\partial u_{k}}{\partial x_{k}}\right)-S_{i j}^{2}\right] \geq 0 \\
i, j=1,2,3, \quad i \neq j
\end{gathered}
$$

The boundary conditions for the model are set as follows.

Inflow: $\quad k_{\infty}=\left(10^{-3} U_{\infty}\right)^{2}, \quad \omega_{\infty}=10^{-4} R e \cdot U_{\infty} / L$

Wall: $\quad k_{\text {wall }}=0, \quad \omega_{\text {wall }}=\frac{6 \mu}{\beta \rho\left(A y_{1}\right)^{2}}$

In Eq. (13), constant $A$ is found to depend on the order of accuracy, or the polynomial degree of the solution in the computational cell using FR. The value of $A=1 / \sqrt{10}$ corresponds to the suggestion made by Menter, ${ }^{11)}$ which is a standard value used for the finite-volume or finite-difference 
scheme. The influence of $A$ is shown in the following section.

\section{Numerical Method}

The essence of the FR approach ${ }^{5,6)}$ and our treatment of curved cells (elements) are briefly described in this section. Each quadrilateral cell in two-dimensional (2D), or hexahedral cell in three-dimensional (3D) CFD grids in physical space, is first transformed to a standard element $\xi \in[-1,1]^{\mathrm{dim}}$ in the computational space using the following mapping,

$$
\boldsymbol{x}=\sum_{k=1}^{m} M_{k}(\boldsymbol{\xi}) \boldsymbol{x}_{k}
$$

where $M_{k}$ is a shape function, $\boldsymbol{x}_{k}$ is a position vector of the cell node and $m$ is the number of nodes composing the cell. Along the curved walls of the wing surfaces, cubic shape functions are used while a linear shape function is used for the flat-plate case shown later. The governing equation, Eq. (1), in the Cartesian coordinate is rewritten on the standard element as follows.

$$
\begin{gathered}
\partial \hat{Q} / \partial t+\partial \hat{F}_{i} / \partial \xi_{i}=\hat{S} \\
\hat{Q}=|J| Q, \quad \hat{F}_{i}=|J| J_{i j}^{-1} F_{j}, \quad \hat{S}=|J| S
\end{gathered}
$$

where $J$ and $|J|$ are the Jacobian matrix of transformation of Eq. (14), $J=\partial(\boldsymbol{x}) / \partial(\boldsymbol{\xi})$, and its determinant, respectively. $J_{i j}^{-1}$ denotes the $(i, j)$ element of $J^{-1}$. Equation (15) holds element-wise, or on each element in the computational domain.

In the FR approach, the solution $\hat{Q}$ and flux $\hat{F}$ are defined at $N$ internal degrees of freedom (DOF) in each direction in the cell. The solution is then expressed by the local Lagrange polynomial of degree $p=N-1$ in the cell, thus the ideal order of accuracy is $p+1$. In Eq. (15), the spatial derivative of the flux is evaluated as a sum of the derivative of the local Lagrange polynomial of the flux and the derivative of the 'flux correction functions.'

$$
\begin{aligned}
\frac{\partial \hat{F}}{\partial \xi}= & \sum_{j=1}^{N} \hat{F}_{j} \cdot \phi_{j}^{\prime}(\xi)+\left[\tilde{F}_{\mathrm{com}}^{-}-\hat{F}_{0}\right] g_{L}^{\prime}(\xi) \\
& +\left[\tilde{F}_{\mathrm{com}}^{+}-\hat{F}_{N+1}\right] g_{R}^{\prime}(\xi)
\end{aligned}
$$

In Eq. (17), $\phi$ is the basis of the Lagrange polynomial of degree $p$ in the $\xi$ direction in the cell. The second and third terms on the right-hand side are corrections required for the flux to be continuous at the cell boundaries by maintaining the common numerical flux $\tilde{F}_{\text {com }}^{\mp}$ with the left/right neighbor cells, which are calculated by an approximate Riemann solver for inviscid flux and a BR2 ${ }^{2)}$ scheme for viscous flux. The Rusanov scheme is used as a Riemann solver in this study. $\hat{F}_{0 / N+1}$ is the extrapolated value by the Lagrange polynomial to the left/right cell boundaries. Finally, $g_{L / R}$ are left/right correction functions of polynomial degree $p+1$, that are one-degree higher than the solution polynomial and satisfy the boundary conditions of $g_{L}(-1)=1$, $g_{L}(1)=0, g_{R}(-1)=0$ and $g_{R}(1)=1$. The location of the

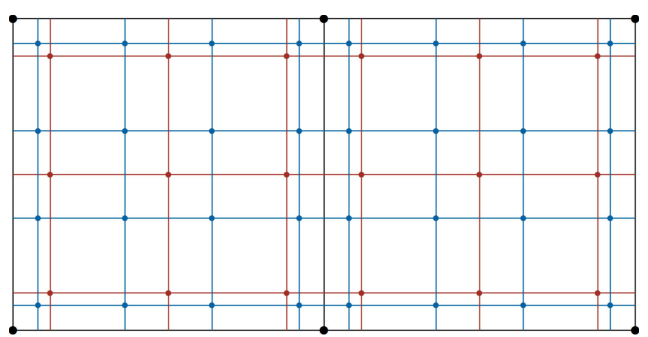

Fig. 1. Base grid and Gauss solution points $(p=2$ and $p=3)$.

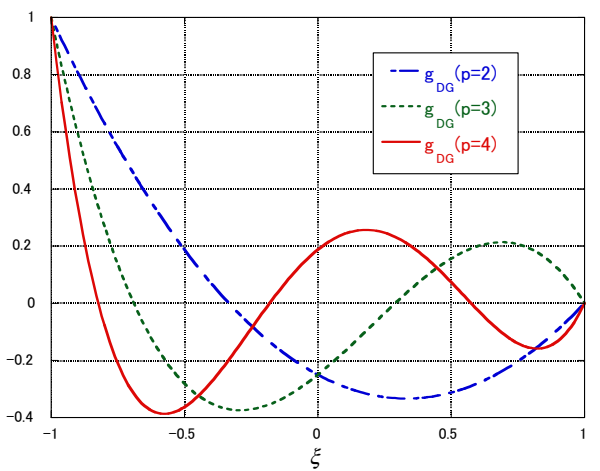

Fig. 2. Radau (DG) correction functions for the left bound for $p=1$ to 3 (corrections for the right bound are symmetrical).

solution points and the correction function determine a specific scheme in the FR. We use Gauss solution points as shown in Fig. 1 and Radau polynomials for the correction as shown in Fig. 2, which are equivalent to the DG, as proved by Huynh. ${ }^{5)}$

It is known that improper evaluation of the transformation metrics $|J| J^{-1}$ of high-order shape functions cannot satisfy the conservation law numerically and cannot preserve the uniform flow. Therefore, special care must be taken when evaluating the metrics. Instead of using Eqs. (15) and (17), we use a simple way to avoid the problem by adopting a modified form derived through constructing a multidimensional Lagrange polynomial of the transformed fluxes, as follows. ${ }^{12)}$ Using explicit notations such as $\hat{F}_{1}=\hat{F}, \hat{F}_{2}=\hat{G}$, $|J| J_{11}^{-1}=\hat{\xi}_{x},|J| J_{12}^{-1}=\hat{\xi}_{y},|J| J_{21}^{-1}=\hat{\eta}_{x},|J| J_{22}^{-1}=\hat{\eta}_{y}$ in 2D for brevity, the fluxes are written as,

$$
\begin{aligned}
\hat{F}(\xi, \eta)= & \hat{F}_{e}(\xi, \eta)+\left[\tilde{F}_{\text {com }}^{-}(\eta)-\hat{F}_{e}(-1, \eta)\right] g_{L}(\xi) \\
& +\left[\tilde{F}_{\text {com }}^{+}(\eta)-\hat{F}_{e}(1, \eta)\right] g_{R}(\xi) \\
= & \hat{F}_{e}(\xi, \eta)+\Delta \hat{F}^{-} \cdot g_{L}(\xi)+\Delta \hat{F}^{+} \cdot g_{R}(\xi) \\
\hat{G}(\xi, \eta)= & \hat{G}_{e}(\xi, \eta)+\left[\tilde{G}_{\mathrm{com}}^{-}(\xi)-\hat{G}_{e}(\xi,-1)\right] g_{L}(\eta) \\
& +\left[\tilde{G}_{\mathrm{com}}^{+}(\xi)-\hat{G}_{e}(\xi, 1)\right] g_{R}(\eta) \\
= & \hat{G}_{e}(\xi, \eta)+\Delta \hat{G}^{-} \cdot g_{L}(\eta)+\Delta \hat{G}^{+} \cdot g_{R}(\eta)
\end{aligned}
$$

where

$$
\hat{F}_{e}(\xi, \eta)=\hat{\xi}_{x} F_{e}+\hat{\xi}_{y} G_{e}, \quad \hat{G}_{e}(\xi, \eta)=\hat{\eta}_{x} F_{e}+\hat{\eta}_{y} G_{e}
$$

and, with $j, k$ respectively denoting DOF in the $\xi, \eta$ direction, 


$$
\begin{aligned}
F_{e} & =\sum_{j}^{N} \sum_{k}^{N} F_{j, k} \phi_{j}(\xi) \phi_{k}(\eta), \\
G_{e} & =\sum_{j}^{N} \sum_{k}^{N} G_{j, k} \phi_{j}(\xi) \phi_{k}(\eta)
\end{aligned}
$$

Substituting Eqs. (18) and (19) in Eq. (15), applying the chain rule and using an analytical identity such as $\partial \hat{\xi}_{x} / \partial \xi+\partial \hat{\eta}_{x} / \partial \eta=0$ and $\partial \hat{\xi}_{y} / \partial \xi+\partial \hat{\eta}_{y} / \partial \eta=0$ results in the final form actually used in this study.

$$
\begin{aligned}
-\frac{\partial \hat{Q}}{\partial t}= & \hat{\xi}_{x} \frac{\partial F_{e}}{\partial \xi}+\hat{\xi}_{y} \frac{\partial G_{e}}{\partial \xi}+\Delta \hat{F}^{-} g_{L}^{\prime}(\xi)+\Delta \hat{F}^{+} g_{R}^{\prime}(\xi) \\
& +\hat{\eta}_{x} \frac{\partial F_{e}}{\partial \eta}+\hat{\eta}_{y} \frac{\partial G_{e}}{\partial \eta}+\Delta \hat{G}^{-} g_{L}^{\prime}(\eta)+\Delta \hat{G}^{+} g_{R}^{\prime}(\eta)+\hat{S}
\end{aligned}
$$

where $\Delta \hat{F}^{ \pm}$and $\Delta \hat{G}^{ \pm}$are defined in Eqs. (18) and (19). With governing Eq. (22), the metrics can be analytically evaluated from Eq. (14) and uniform flow is obviously preserved. A main problem of the non-conservative form in Eq. (22) is that an exact volume integral of the right-handside flux is not obtained by numerical integration using discrete values at the solution points, and thus, this form no longer satisfies discrete conservation in general. Our results in the later section do not show noticeable defects despite this problem. A strict evaluation of the conservation property by comparison with a fully conservative form ${ }^{13)}$ will be conducted in future work.

For high-Reynolds number RANS simulations with small cell sizes near the wall, implicit time integration is effective to relax the severe restriction for the time step and advance the solution $\hat{Q}$ to the steady-state. The Block LU-SGS ${ }^{8)}$ implicit method is used in this study. The RANS and $k$ - $\omega$ equations are fully coupled and advanced in time simultaneously.

\section{Results and Discussions}

Results for three problems are shown in this section. They are flat-plate boundary layer, NACA4412 airfoil trailingedge separation and the three-element NHLP/L1T2 HLD. A $3 \mathrm{D}$ flow solver is used to simulate the $2 \mathrm{D}$ flows above. The computational grid is extruded in the span-wise direction to have only one cell in that direction. Single-block or multiblock structured grids are converted to unstructured data sets to be used in the flow solver. Total DOF in this section are counted as two-dimensional problems.

\subsection{Verification of the $k-\omega$ turbulence model for the FR method for a flat-plate boundary layer}

The basic performance and properties of the turbulence model for the FR method are verified using a flat-plate boundary layer problem. The freestream Mach number is 0.2 and Reynolds number per unit length is $\operatorname{Re}=10^{7}$. The number of cells in the 'base grid' is $23 \times 20$ and the nondimensional distance to the first grid point off the wall (the grid cell size attached to the wall) is $0.02 / \sqrt{R e} \approx 6.3 \times 10^{-6}$, except for one case shown as 'fine' in Figs. 3(a)-(c). The

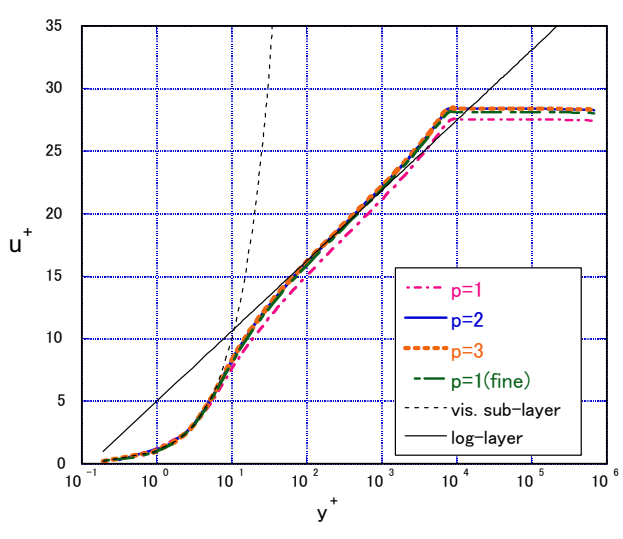

(a) Velocity

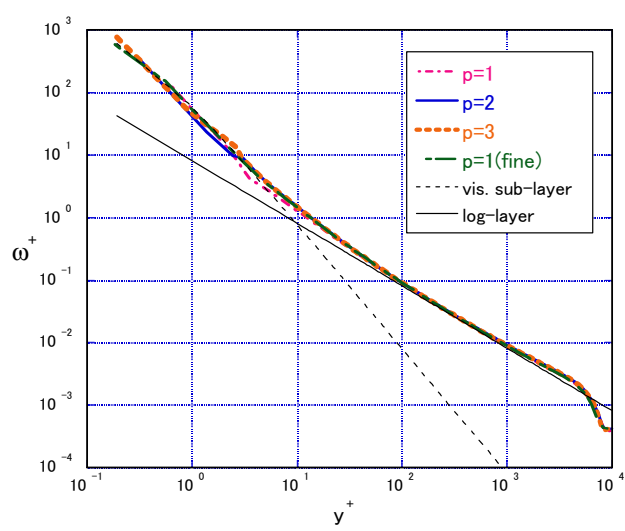

(b) Specific dissipation rate

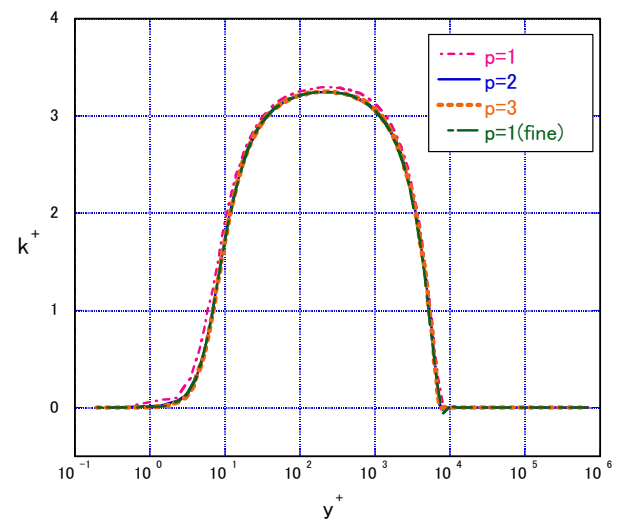

(c) Turbulent kinetic energy

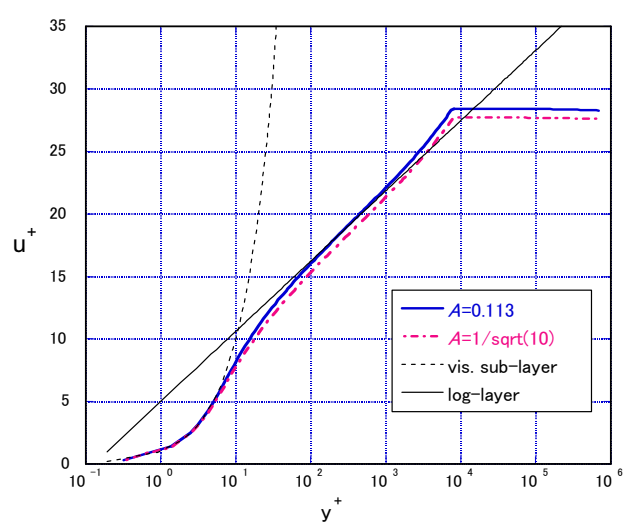

(d) Effect of the wall-boundary condition of $\omega(p=2)$

Fig. 3. Property of the flat-plate boundary layer in the wall unit. 
polynomial degrees of the solutions in this problem are $p=1$ (second-order accuracy), $p=2$ (third-order) and $p=3$ (fourth-order). Thus, the total DOF are 1,840, 4,140 and 7,360, respectively. The high-order simulations were efficiently run using the interpolated initial solution from the lower-order solution. Figures 3(a)-(c) show the profiles of computed velocity $u^{+}$, specific dissipation rate $\omega^{+}$and turbulent kinetic energy $k^{+}$in the wall unit. The $u^{+}$and $\omega^{+}$are compared with the law of the wall in Figs. 3(a) and (b). The law of the wall in terms of the dissipation rate is expressed as follows.

$$
\begin{gathered}
\omega^{+}=\omega_{\text {wall }}^{+} /\left(y^{+} \sqrt{\beta \omega_{\mathrm{wall}}^{+} / 6}+1\right)^{2}: \text { viscous sublayer } \\
\omega^{+}=1 / \sqrt{\beta^{*}} \kappa y^{+}: \text {log-law }
\end{gathered}
$$

where $\kappa=0.41$ is the Karman constant and other constants are in Eq. (9). In Fig. 3(c), $k^{+}$takes a peak value in the middle of the inertia region at $y^{+}=10^{2}-10^{3}$. Figures $3(\mathrm{a})-(\mathrm{c})$ also show the importance of the minimum cell size. The case ' $p=1$ ' uses a common base grid as $p=2$ and 3 , but the result does not agree well with the velocity profile in the loglayer. In the case of ' $p=1$ (fine), the minimum cell size is reduced by a factor of 0.25 while the total number of grid points is unchanged. The result is improved and agrees well with the results for $p=2$ and 3 . We had a similar experience in simulations of high-lift devices in the following subsection. Optimal base grids for higher degrees of polynomial were too coarse for $p=1$. From these observations, we conclude that the distance from the wall surface to the first solution point (not cell size) must be about $0.005 / \sqrt{R e}$ independent of polynomial degree.

The value of constant $A$ in Eq. (13) for the wall boundary condition of $\omega$ is determined depending on polynomial degrees as suggested for the DG reported by Bassi et al. ${ }^{2)}$ or Schoenawa et al. ${ }^{3)}$ They estimated the value by approximating the behavior of the near-wall analytical solution, $\omega=6 \mu / \beta \rho y^{2}$, using Taylor series expansion or $\mathrm{L}^{2}$-projection onto the basis of the orthogonal polynomial space. The values obtained for $A$ are rather different for each method, indicating the need for further discussion as well as the importance of actual calibrations. In our implementation using FR, we found a simple and rational way. In order to effectively scale the grid resolution depending on the polynomial degree, the value ' $A y_{1}$ ' in Eq. (13) is set equal to the distance from the wall surface to the first solution point above the wall. Therefore, in a standard element $\xi \in[-1,1]$, ' $A$ ' is half the distance from the lower boundary $(\xi=-1)$ to the first solution point. The constant $A$ for Gauss-solution points is shown in Table 1, which is about two- to threetimes larger than that in Schoenawa et al. ${ }^{3)}$

Table 1. Constant ' $A$ ' for wall-boundary conditions of $\omega$.

\begin{tabular}{ccc}
\hline$p=1$ & $p=2$ & $p=3$ \\
\hline 0.211 & 0.113 & 0.0694 \\
\hline
\end{tabular}

The effect of the above boundary condition is shown in Fig. 3(d) for $p=2$. The result with standard value $A=1 / \sqrt{10}$ is not as accurate as that with the modified value, as shown in Table 1.

\subsection{High angle-of-attack NACA4412}

The second verification is a high angle-of-attack single airfoil with adverse pressure gradients and small flow separations. The case for NACA4412 was reported in Turbulence Modeling Resources ${ }^{10)}$ and by Menter. ${ }^{11}$ ) The freestream Mach number is 0.2 , Reynolds number is $1.52 \times 10^{6}$ based on the chord and the angle of attack is 13.85 degrees. The number of cells for the base grid is $86 \times 45$. The results of $p=2$ with 34,830 DOF is shown here. Figures 4(b) and (c) show distributions of the velocity and the Reynolds stress, respectively, along three broken-lines shown in Fig. 4(a). The three cross-sections near the trailing-edge are $x / c=0.7308$, 0.8418 and 0.9528 . Although the extent of the reversed flow shows some discrepancy from the experiment due to the

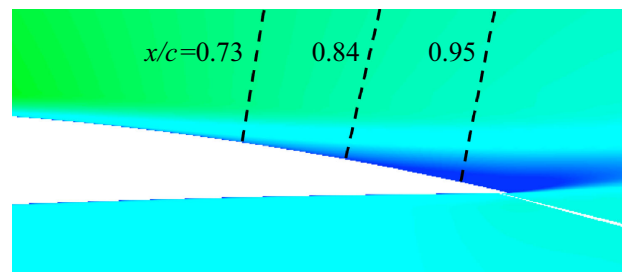

(a) Mach contours near the trailing-edge

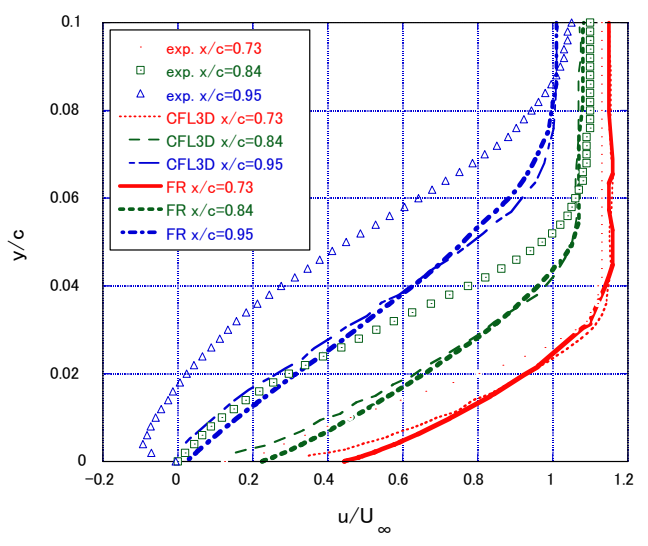

(b) Velocity distributions

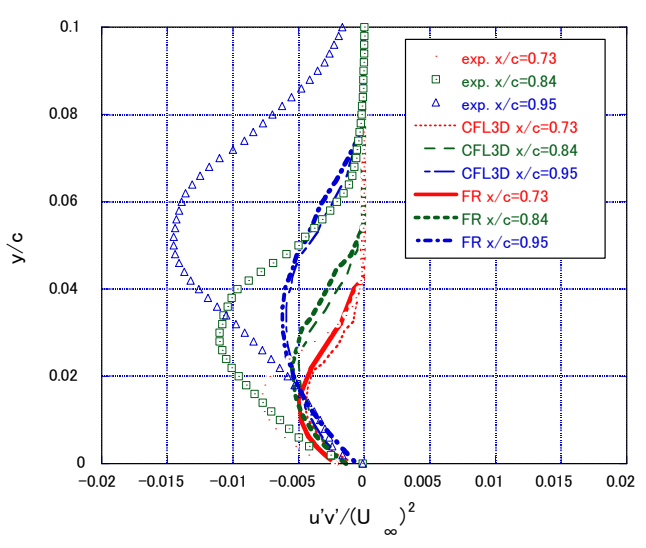

(c) Reynolds stress distributions

Fig. 4. NACA4412 at $\mathrm{AOA}=13.85 \mathrm{deg}$ and $R e=1.52 \times 10^{6}$. 


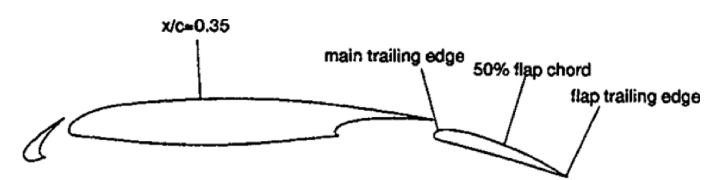

Fig. 5. Configurations and locations of total-pressure measurements.

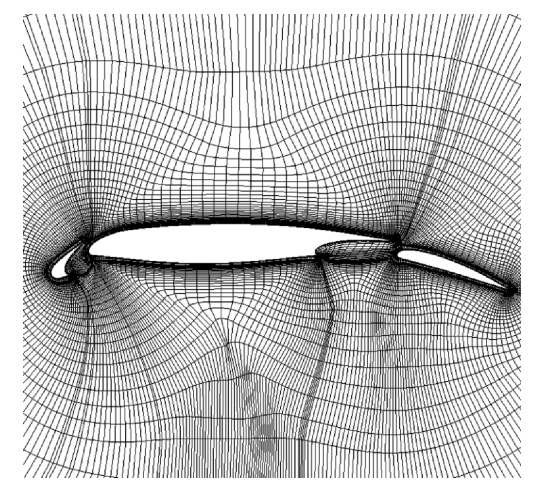

Fig. 6. Base grid (standard) with 17,079 cells.

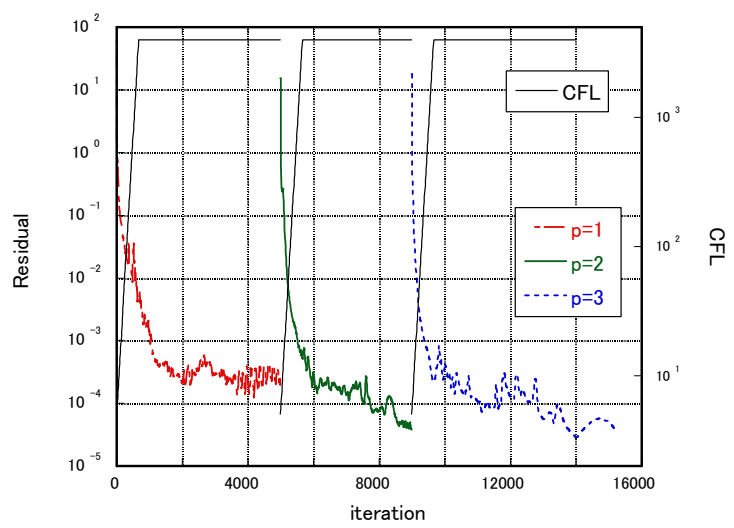

Fig. 7. Convergence histories for three-element wing.

property of the $k$ - $\omega$ model, both Figs. 4(b) and (c) show good agreement with reference CFD data ${ }^{10)}$ obtained using a thirdorder upwind-biased finite difference code (CFL3D). The cross-code verification for this sensitive case confirms that the model has been properly implemented in the FR scheme.

\subsection{Three-element airfoil}

The main objective of the study is the case referred as NHLP/L1T2, which offers elaborate experimental data as the result of using a wind tunnel ${ }^{14)}$ and is a well-known test case for the CFD. ${ }^{15,16)}$ A $2 \mathrm{D}$ airfoil is separated into three elements, and the deflection angles of the slat and flap are 25 and 20 degrees, respectively, to simulate landing conditions. The freestream Mach number is 0.197 , the Reynolds number based on the original chord is $3.25 \times 10^{6}$ and the angle-of-attack is 20.18 degrees. The configuration and locations of the total pressure measurement are shown in Fig. 5. In the experiment, surface pressures are also measured and the lift and drag forces are calculated by integrating the pressure over the surface. In this study, surface pressure coefficients and total pressure profile in the wake are compared to experimental results. The standard base grid for the com-

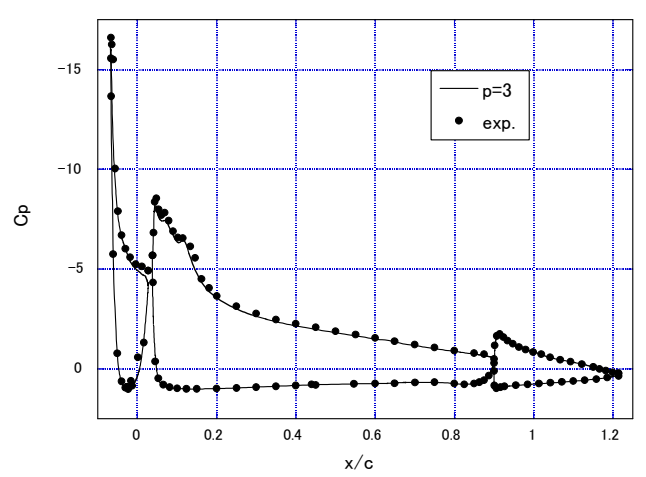

(a) Overall comparison of $p=3$ results and the experiment

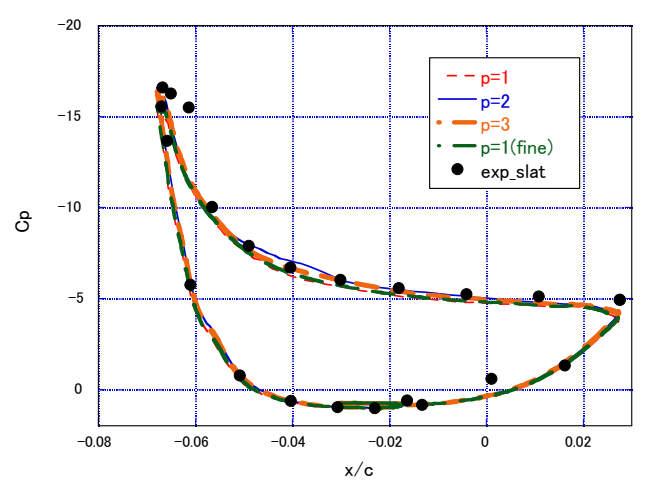

(b) Slat

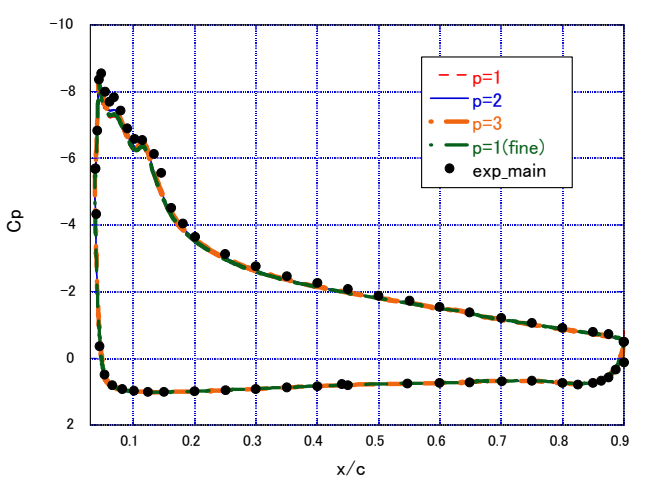

(c) Main wing

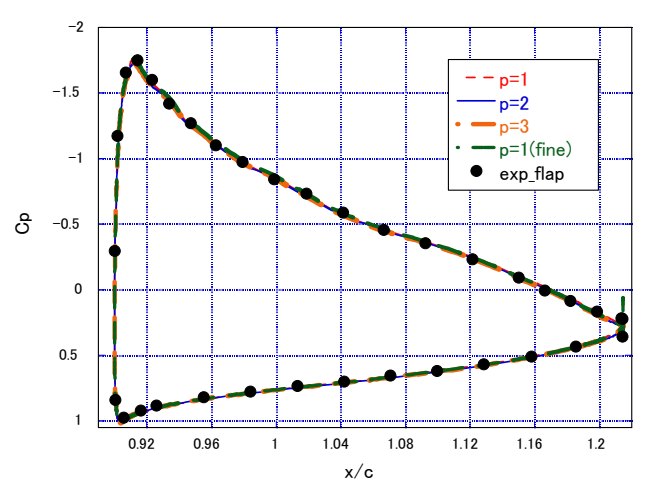

(d) Flap

Fig. 8. Comparisons of $C_{p}$ distributions.

putation is shown in Fig. 6. The total number of cells is 17,079 and the multi-block structured grids are converted to single-block unstructured grids. The size of the whole computational domain is approximately 100 times as large 
Table 2. Lift and drag forces.

\begin{tabular}{lll}
\hline & $C_{\mathrm{L}}$ & $C_{\mathrm{D}}$ \\
\hline Exp. & 4.07 & 0.0720 \\
$p=3$ & 4.06 & 0.0761 \\
\hline
\end{tabular}

as the chord length. Four simulation results are shown, two of which use a common base grid for the polynomial degrees of $p=2$ and 3. As is reported for the flat-late case in Section 4.1 , the minimum cell size on the wall is too coarse for $p=2$. Therefore, it is reduced by a factor of 0.25 and the grid points are redistributed without changing the number of grid points. The other case is the $p=1$ simulation on a base grid nearly twice as fine in every direction, resulting in 65,734 cells. The minimum cell size of the fine grid is the same as the reduced one on the standard base grid for $p=1$.

At first, convergence histories from $p=1$ to 3 are shown in Fig. 7. The initial conditions for high-order accurate simulations are interpolated solutions from the lower-order solution. The histories of CFL numbers are also plotted. Starting from $\mathrm{CFL}=5$, it is multiplied by an amplification factor of 1.01 at every time step and increases to the upper bound of $\mathrm{CFL}=4,000$. The implicit time integration described in Section 3 is robust, even for the turbulence model at the cost of numerical computations of the large sparse Jacobian matrix for the implicit method. Rough estimations of the cost per DOF in terms of the ratio of the calculation time to that of our implicit finite-volume code are 3 for $p=1,7.5$ for $p=2$, and 9 for $p=3$.

Figures 8(a)-(d) show the distributions of the surface pressure coefficients. Figure 8(a) shows the overall comparisons of the $p=3$ simulations and the experiment. Figures $8($ b)-(d) also show the pressure coefficients on each element and compare the orders of accuracy. All four solutions are very close to each other and did well reproducing the leading-edge suction peak on the upper surface in the experiment. The $C_{p}$ distributions downstream also agree well with the experiment for all of the elements. We note here that reducing the minimum cell size of the base grid only for the $p=1$ simulation had a large effect on the solution. In the preliminary results, only the $p=1$ solution could not capture the suction peak and the predicted pressure was higher everywhere.

The lift and drag coefficients for the case of $p=3$ are compared in Table 2. In the experiment, they were obtained by integrating the pressure coefficient.

Figures 9(a)-(d) show the distributions of total pressure along the four lines shown in Fig. 5. In Fig. 9(a) at $x / c=0.35$ above the main wing, the distinct minimum value is the total pressure loss due to the wake of the slat. In Figs. 9(b)-(d), two minima in the $C_{p 0}$ are observed, the lower one of which corresponds to the total pressure loss due to the main wing. Unlike the almost identical results for surface $C_{p}$ plots, differences are observed among the simulations. The results using $p=2$ and 3 show remarkable improvements against $p=1$. They are close to each other and reproduce the experimental results very well. The improvement using

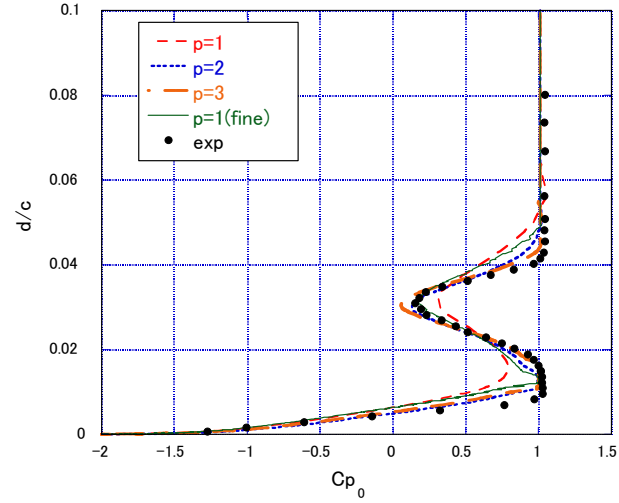

(a) $x / c=0.35$ above the main wing

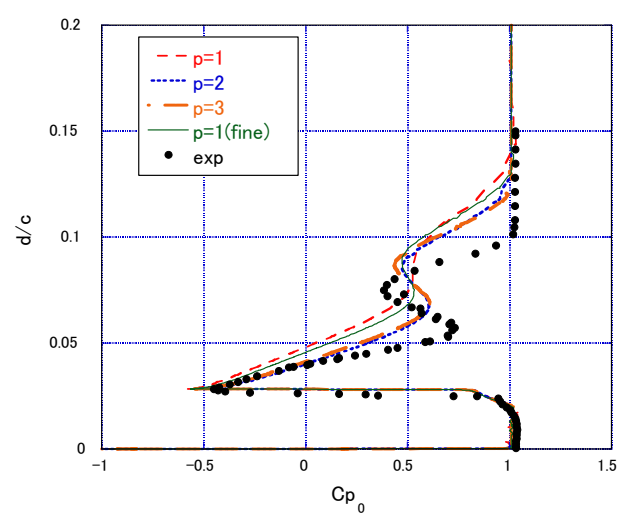

(b) Main trailing-edge

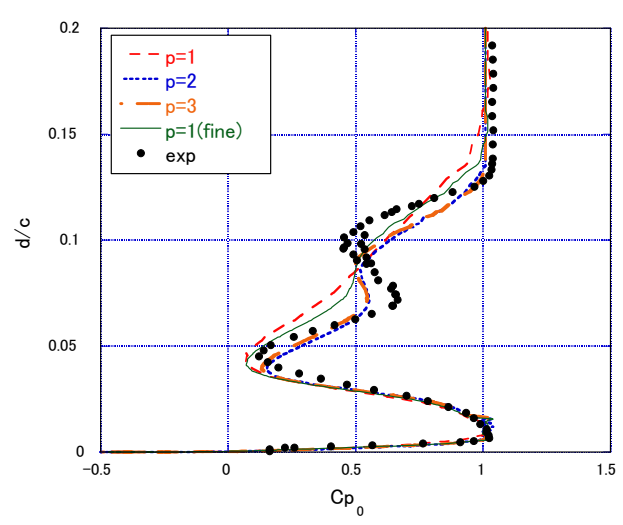

(c) $50 \%$ flap chord

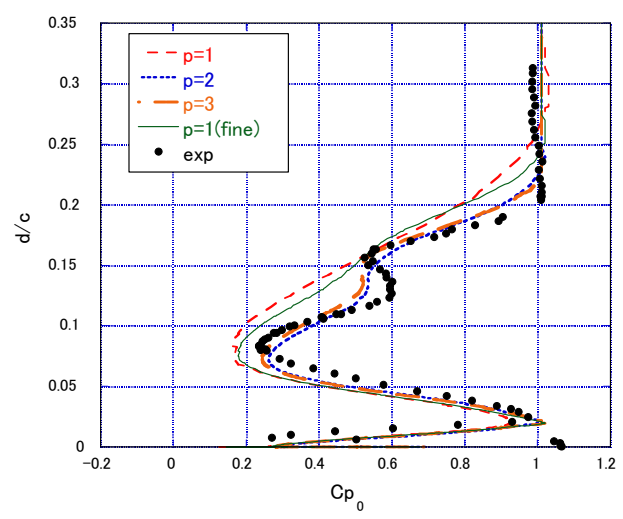

(d) Flap trailing-edge

Fig. 9. Total pressure coefficients: influence of spatial accuracy. 


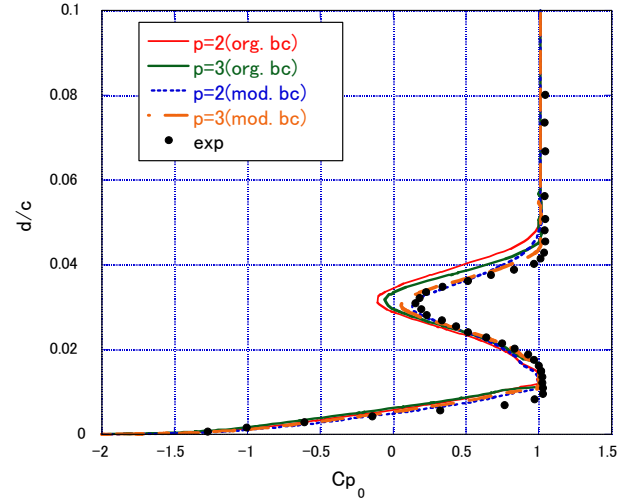

(a) $x / c=0.35$ above the main wing

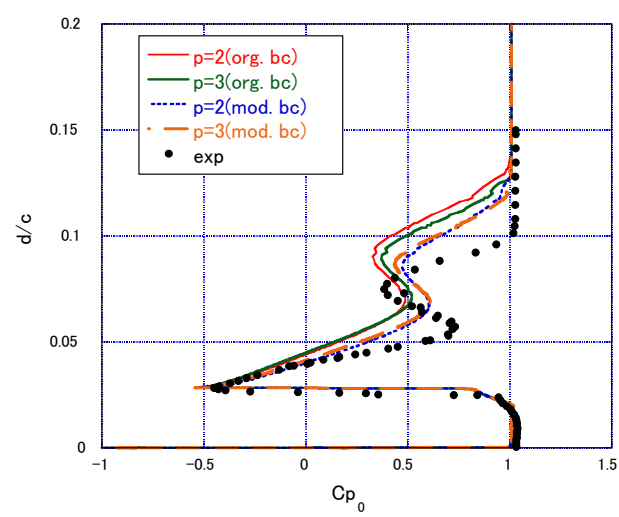

(b) Main trailing-edge

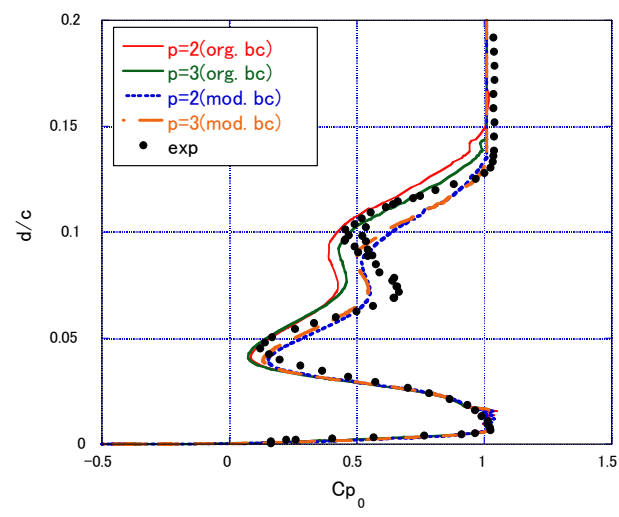

(c) $50 \%$ flap chord

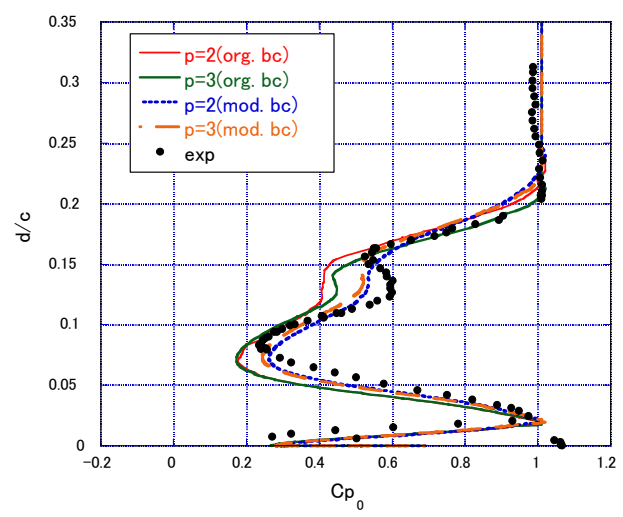

(d) Flap trailing-edge

Fig. 10. Total pressure coefficients: influence of the boundary conditions. the fine base grid is also clear in Figs. 9(a) and (b), where the result on the fine grid is closer to $p=2$ and 3 results. However the wake of the slat diffuses downstream and the difference between the two base grids for $p=1$ becomes smaller in Figs. 9(c) and (d).

The effect of the wall boundary condition of $\omega$ is again investigated in Figs. 10(a)-(d). The results using the standard value of $A=1 / \sqrt{10}$ are compared with the results of the modified values already shown in Figs. 9(a)-(d). The appropriate boundary condition better captures the wake profile at every section. The effect of the boundary condition inside the boundary layer looks small in Figs. 10(a), (c) and (d) due to thin boundary layers at the forepart of the main wing and the short flap. On the other hand, for the boundary layer developed at the trailing-edge of the main wing in Figs. 10(b), distinct differences appear in the region around $y=0.028$ to 0.065 , which then form the wake profile downstream in Figs. 10(c) and (d).

\section{Conclusion}

The flow solution method for the RANS and $k-\omega$ turbulence mode is developed using a high-order FR scheme. We found numerical techniques similar to the DG are mandatory for the FR as well. They are transformation of the specific dissipation rate, realizability conditions for the Reynolds stresses, and modified boundary conditions depending on the order of accuracy (polynomial degree). The impact of high-order accuracy was clearly shown for the analysis of HLD. This study provides an important step for high-order CFD to be incorporated in the analysis and design of aircraft aerodynamics.

\section{Acknowledgments}

This work was supported by JSPS KAKENHI Grant Number $15 \mathrm{~K} 14248$.

\section{References}

1) Murayama, M., Yamamoto, K., Kentaro, T., and Ueda, Y.: CFD Analysis of JAXA's High-Lift Wind-Tunnel Model, Proceedings of 44th Aircraft Symposium, 2B7, CD-ROM, 2006 (in Japanese).

2) Bassi, F., Crivellini, A., Rebay, S., and Savini, M.: Discontinuous Galerkin Solution of the Reynolds-averaged Navier-Stokes and k-w Turbulence Model Equations, Comput. Fluids, 34 (2005), pp. 507-540.

3) Schoenawa, S., Hartmann, R., Rebay, S., and Savini, M.: Discontinuous Galerkin Discretization of the Reynolds-averaged Navier-Stokes Equations with the Shear-stress Transport Model, J. Comput. Phys., 262 (2014), pp. 194-216.

4) Liang, C., Jameson, A., and Wang, Z. J.: Spectral Difference Method for Compressible Flow on Unstructured Grids with Mixed Elements, J. Comput. Phys., 228 (2009), pp. 2847-2858.

5) Huynh, H. T.: A Flux Reconstruction Approach to High-Order Schemes Including Discontinuous Galerkin Methods, AIAA Paper 2007-4079, 2007.

6) Huynh, H. T.: A Reconstruction Approach to High-Order Schemes Including Discontinuous Galerkin for Diffusion, AIAA Paper 2009-403, 2009.

7) Miyaji, K.: Vortical Flow Simulations by a High-order Accurate 
Trans. Japan Soc. Aero. Space Sci., Vol. 60, No. 1, 2017

Unstructured Hexahedral Grid Method, Comput. Fluids, 85 (2013), pp. 78-84.

8) Skarolek, V. and Miyaji, K.: Numerical Simulation of Transitional Flow Past a Wing Using Flux Reconstruction Method, AIAA J., 54, 4 (2016), pp. 1424-1437.

9) Wilcox, D. C.: Reassessment of the Scale-Determining Equations for Advanced Turbulence Models, AIAA J., 26, 11 (1988), pp. 1299-1310.

10) Turbulence Modeling Resources, http://turbmodels.larc.nasa.gov (accessed October 3, 2015)

11) Menter, F. R.: Two-Equation Eddy-Viscosity Turbulence Models for Engineering Applications, AIAA J., 32, 8 (1994), pp. 1598-1605.

12) Liang, C., Miyaji, K., and Zhang, B.: An Efficient Correction Procedure via Reconstruction for Simulation of Viscous Flow on Moving and Deforming Domains, J. Comput. Phys., 256 (2014), pp. 55-68.
13) Abe, Y., Haga, T., Nonomura, T., and Fujii, K.: On the Freestream Preservation of High-order Conservative Flux-reconstruction Scheme, J. Comput. Phys., 281 (2015), pp. 28-54.

14) Moir, I. R. M.: Measurements on a Two-Dimensional Aerofoil with High-Lift Devices, AGARD Advisory Report No. 303, Vol. 2, 1994, pp. A2-1-A2-12.

15) Fejtek, I.: Summary of Code Validation Results for a Multiple Element Airfoil Test Case, AIAA Paper 97-1932, 1997.

16) Murayama, M., Lei, Z., Mukai, J., and Yamamoto, K.: CFD Validation for High-Lift Devices: Three-Element Airfoil, Trans. Jpn. Soc. Aeronaut. Space Sci., 49 (2006), pp. 40-48.

K. Sawada

Associate Editor 